\title{
CMB polarization towards clusters as a probe of the integrated Sachs-Wolfe effect
}

\author{
Asantha Cooray* \\ California Institute of Technology, Mail Code 130-33, Pasadena, California 91125 \\ Daniel Baumann ${ }^{\dagger}$ \\ California Institute of Technology, Mail Code 130-33, Pasadena, California 91125 \\ and University of Cambridge, Cambridge, CB3 OHE, United Kingdom \\ (Received 26 October 2002; published 17 March 2003)
}

\begin{abstract}
The scattering of the temperature anisotropy quadrupole by free electrons in galaxy clusters leads to a now well-known polarization signal in the cosmic microwave background fluctuations. Using multifrequency polarization data, one can extract the temperature quadrupole and separate it from the contaminant polarization associated with the kinematic quadrupole due to the transverse motion of clusters. At low redshifts, the temperature quadrupole contains a significant contribution from the integrated Sachs-Wolfe effect (ISW) associated with the growth of density fluctuations. Using polarization data from a sample of clusters over a wide range in redshift, one can statistically establish the presence of the ISW effect and determine the redshift dependence of the ISW contribution to the rms quadrupole. Given the strong dependence of the ISW effect on the background cosmology, the cluster polarization can eventually be used as a probe of the dark energy.

DOI: 10.1103/PhysRevD.67.063505

PACS number(s): 98.80.Es, 95.85.Nv, 98.35.Ce, 98.70.Vc
\end{abstract}

\section{INTRODUCTION}

Linear polarization of the cosmic microwave background $(\mathrm{CMB})$ is generated through rescattering of the temperature quadrupole [1]. At the last scattering surface, this leads to the primordial polarization contribution [2], while at lower redshifts, the temperature quadrupole gets scattered in regions containing free electrons to produce a secondary polarization signal. One such possibility arises from reionization which generates a large angular scale polarization contribution corresponding to the horizon at the surface of reionization [3]. At much smaller angular scales corresponding to a few arcminutes and below, secondary polarization is generated when the quadrupole is scattered by free electrons in galaxy clusters [4].

There are several mechanisms to generate a polarization signal in clusters. However, scattering of the primordial temperature anisotropy quadrupole is expected to dominate the total contribution $[5,6]$. A second effect arises from the kinematic quadrupole due to the transverse motion of the cluster. This contribution, fortunately, has a different frequency dependence than the contribution associated with the scattering of the primordial quadrupole.

Following initial suggestions on the cluster polarization contributions [4], recent studies have focused on detailed aspects related to spectral dependences $[5,6]$, the statistical signature such as the angular power spectra of polarization [7], and the use of cluster polarization as a mechanism to improve the local determination of the temperature quadrupole [8].

Here, we suggest an extension to the discussion in Ref. [8]. The basic idea is to use galaxy clusters as a tracer of the temperature anisotropy quadrupole and to statistically detect

\footnotetext{
*Email address: asante@tapir.caltech.edu

${ }^{\dagger}$ Email address: db275@tapir.caltech.edu
}

its rms value. Our discussion involves the measurement of the quadrupole as a function of redshift or redshift bins based on a cluster catalogue. We improve the previous discussion in Ref. [8] by considering the contaminant kinematic quadrupole contribution and introducing the use of multifrequency observations. We make a quantitative prediction for the prospects of measuring the redshift evolution of the temperature quadrupole with future $\mathrm{CMB}$ polarization experiments.

The statistical reconstruction of the rms temperature quadrupole, as a function of redshift, is useful for several purposes. At low redshifts, the quadrupole contains information related to growth of structures via the integrated SachsWolfe (ISW) effect [9]. The presence of the ISW effect, however, cannot be established from the temperature anisotropy measurements alone, due to the dominant Sachs-Wolfe (SW) contribution and the large sample variance associated with low multipoles.

Since the ISW contribution mainly arises from low redshifts, one expects large scale CMB contributions to be correlated with maps of the large scale structure [10]. This correlation, however, is hard to establish observationally due to both large cosmic variance and the lack of adequate signalto-noise all-sky maps of tracer fields [11]. One therefore needs a probe of the ISW effect that is not subject to a large sample variance and we propose the use of cluster polarization for this purpose. Using the polarization signal towards a sample of clusters widely distributed over the whole sky, one can establish the presence of the ISW effect reliably. Additionally, since the ISW contribution is strongly sensitive to the background cosmology, the redshift evolution of the rms quadrupole can be used as a probe of the dark energy.

The paper is organized as follows. In Sec. II, we introduce polarization signals towards clusters associated with the scattering of the primordial $\mathrm{CMB}$ temperature quadrupole and with the kinematic quadrupole generated by transverse cluster motions and study the use of multi-frequency polarization 
observations as a way to separate these two contributions. We will then consider the reconstruction of the rms temperature quadrupole as a function of redshift using polarization observations towards a sample of galaxy clusters. We discuss our results in Sec. III.

\section{POLARIZATION TOWARDS CLUSTERS}

CMB polarization towards clusters is generated when the incident radiation has a nonzero quadrupole moment. The two dominant origins for this quadrupole moment are (a) a quadrupole from primordial CMB fluctuations, and (b) a quadrupole from the quadratic term in the Doppler shift if the scattering gas has a transverse velocity [5-7]. Towards a sufficiently large sample of galaxy clusters, we can write the total rms polarization contribution as [5]

$$
\begin{gathered}
P_{\text {tot }}^{2}=P_{\text {prim }}^{2}+P_{\text {kin }}^{2}, \\
P_{\text {prim }}=\frac{\sqrt{6}}{10}\langle\tau\rangle \frac{Q^{\mathrm{rms}}(z)}{T_{\mathrm{CMB}}}, \\
P_{\text {kin }}=\frac{1}{10} g(x)\langle\tau\rangle\left\langle\beta_{t}^{2}\right\rangle,
\end{gathered}
$$

where $\tau$ is the optical depth to scattering in each cluster: $\tau$ $=\sigma_{T} \int d y n_{e}(y)$. For an individual cluster, the optical depth can be constrained through the temperature fluctuation associated with the Sunyaev-Zel'dovich (SD) [4] effect. Since we are averaging over large samples of clusters, in Eq. (1), we have considered the sample averaged optical depth, $\langle\tau\rangle$. $\beta_{t}$ $=v_{t} / c$ gives the transverse component of the cluster velocity and $g(x)$, with $x=h \nu / k_{B} T_{\mathrm{CMB}}$, is the frequency dependence of the kinematic effect (discussed below).

Note that the rms quadrupole of temperature anisotropies, as a function of redshift, is related to the $l=2$ contribution to the CMB power spectrum as measured by an observer at that redshift: $Q_{\mathrm{rms}}^{2}(z)=5 C_{2}(z) / 4 \pi$. Large angular scale observations, such as from the Cosmic Background Explorer $(\mathrm{COBE})$, constrain the variance of the temperature quadrupole today to be $C_{2}(z=0)=(27.5 \pm 2.4 \mu \mathrm{K})^{2}$, assuming a power-spectrum tilt of unity [12]. The quadrupole is highly uncertain due to the fact that one is limited to only five independent samples. Also, these observations do not allow a determination of the rms quadrupole at redshifts other than today's. However, measuring the CMB polarization towards galaxy clusters potentially allows one to probe the quadrupole moment at the cluster position [8].

We can calculate the expected redshift evolution of the quadrupole based on the two sources of contributions, one at the surface of last scattering due to the SW effect and another at late times due to the ISW effect associated with a time evolving potential [9]. We write these two contributions to the power spectrum, projected to a redshift $z$, as

$$
C_{l}(z)=C_{l}^{\mathrm{SW}}(z)+C_{l}^{\mathrm{ISW}}(z)
$$

$$
\begin{aligned}
C_{l}^{\mathrm{SW}}(z)= & \frac{\Omega_{m}^{2} H_{0}^{4}}{2 \pi a^{2}\left(r_{\mathrm{rec}}\right)} \int_{0}^{\infty} \frac{d k}{k^{2}} P\left(k, r_{\mathrm{rec}}\right) j_{l}^{2}\left[k\left(r_{\mathrm{rec}}-r_{z}\right)\right] \\
C_{l}^{\mathrm{ISW}}(z)= & \frac{18 \Omega_{m}^{2} H_{0}^{4}}{\pi} \int_{0}^{\infty} \frac{d k}{k^{2}} P(k, 0) \\
& \times\left[\int_{r_{z}}^{r_{r e c}} d r_{z}^{\prime} \frac{d}{d r_{z}^{\prime}}\left(\frac{G}{a}\right) j_{l}\left[k\left(r_{z}^{\prime}-r_{z}\right)\right]\right]^{2} .
\end{aligned}
$$

Here, $r_{z}$ is the radial comoving distance out to redshift $z, r_{\text {rec }}$ is the distance to last scattering $(z=1100)$, and $G\left(r_{z}\right)$ is the growth rate of linear density fluctuations with $\delta^{\text {lin }}\left(k, r_{z}\right)$ $=G\left(r_{z}\right) \delta^{\operatorname{lin}}(k, 0)$ [13]. Here, $\delta^{\operatorname{lin}}\left(k, r_{z}\right)$ is the fluctuation in the linear density field and $P\left(k, r_{z}\right)$, in Eq. (2), is its power spectrum.

In addition to scattering via the temperature anisotropy quadrupole, the kinematic quadrupole associated with the transverse motion also produces a polarization signal towards galaxy clusters [4-6]. To understand how this polarization contribution is generated, first consider electrons moving with peculiar velocity, $\beta=v / c$, relative to the rest frame defined by the CMB. The CMB spectral intensity in the mean electron rest frame is Doppler shifted:

$$
I_{\nu}=C \frac{x^{3}}{e^{x \gamma(1+\beta \mu)}-1}
$$

where $x \equiv h \nu / k_{B} T_{C M B}$ and $\mu$ is the cosine of the angle between the cluster velocity and the direction of the incident CMB photon. When expanded in Legendre polynomials, the intensity distribution is

$$
I_{\nu}=C \frac{x^{3}}{e^{x}-1}\left[I_{0}+I_{1} \mu+\frac{e^{x}\left(e^{x}+1\right)}{2\left(e^{x}-1\right)^{2}} x^{2} \beta^{2}\left(\mu^{2}-\frac{1}{3}\right)+\ldots\right]
$$

and contains the necessary quadrupole under which scattering generates a polarization contribution with Stokes parameters given by $Q\left(\mu^{\prime}\right)=1 / 10 g(x) \beta^{2}\left(1-\mu^{\prime}\right)^{2}$ $=1 / 10 g(x) \beta_{t}^{2}, U\left(\mu^{\prime}\right)=0$ where $\mu^{\prime}$ is now the angle between the cluster velocity and the line of sight.

The CMB intensity, $I(x)$, and polarization $P_{\text {prim }}(x)$, due to the temperature quadrupole, have a spectral dependence given by the standard blackbody spectrum, with fractional polarization in temperature units related to that in intensity given by

$$
f(x)=\frac{x e^{x}}{e^{x}-1}
$$

The fractional kinematic polarization, in intensity, has a spectral dependence given by

$$
P_{\text {kin }}(x) \propto \frac{e^{x}\left(e^{x}+1\right)}{2\left(e^{x}-1\right)^{2}} x^{2}
$$


Instead of working in intensity units, we will follow the usual approach and consider separations based on temperature units. Thus, we write the spectral dependence associated with the kinematic quadrupole, relative to CMB thermal temperature, as

$$
g(x)=\frac{x}{2} \operatorname{coth}\left(\frac{x}{2}\right)
$$

Note that the spectral dependence of the kinematic quadrupole contribution gives a potential method to separate the two contributions. This is similar to component separation suggestions in the literature as applied to temperature observations, such as the separation of the SZ thermal effect from dominant primordial fluctuations [14].

\section{A. Frequency separation}

Here, we will consider separation based on real space data instead of the multipolar analysis considered for the temperature [15]. We will briefly discuss the separation problem in the presence of noise. We refer the reader to Ref. [16] for further details. In the present case, the problem involves the extraction of the polarization associated with the primordial quadrupole, with the kinematic quadrupole considered as a source of confusion. We can write the total polarization towards a sample of clusters within a redshift bin as

$$
\mathbf{P}=\mathbf{Q}+\mathbf{K}+\mathbf{N},
$$

where $\mathbf{Q}$ is the contribution due to the primordial quadrupole, $\mathbf{K}$ is the contribution due to the kinematic quadrupole and $\mathbf{N}$ characterizes the instrumental noise. The twodimensional vector field associated with polarization can be decomposed into two scalar quantities involving two orthogonal measurements, which we will simply denote as $\left(P_{x}, P_{y}\right)$. These orthogonal quantities can be related to the usual Stokes parameters involving $Q$ and $U$ or the gradient and curl representation of the spin-2 field involving $E$ and $B$ modes [17].

We assume Gaussian noise with

$$
\begin{gathered}
\left\langle N_{x}\right\rangle=0, \\
\left\langle N_{x}^{a} N_{x}^{b}\right\rangle=C_{a b}^{x},
\end{gathered}
$$

and similarly for the $y$ component. Here, $a, b=1, \ldots, N_{\mathrm{ch}}$ label the different frequency channels of the experiment.

In Fig. 1, we illustrate the frequency dependence of the primordial and kinematic quadrupole contributions using allsky maps of the expected polarization. In these plots, each polarization vector, shown as lines, should be considered as a representation of the polarization towards a cluster at that location. The polarization pattern due to temperature quadrupole scattering is uniform and traces the underlying temperature quadrupole distribution. In describing the kinematic quadrupole we assume, for illustration purposes, a transverse velocity field with $\left\langle\beta_{t}\right\rangle \sim 10^{-3}$ corresponding to a velocity of $300 \mathrm{~km} \mathrm{sec}^{-1}$. The polarization contribution due to the kinematic quadrupole, however, is random because transverse ve-
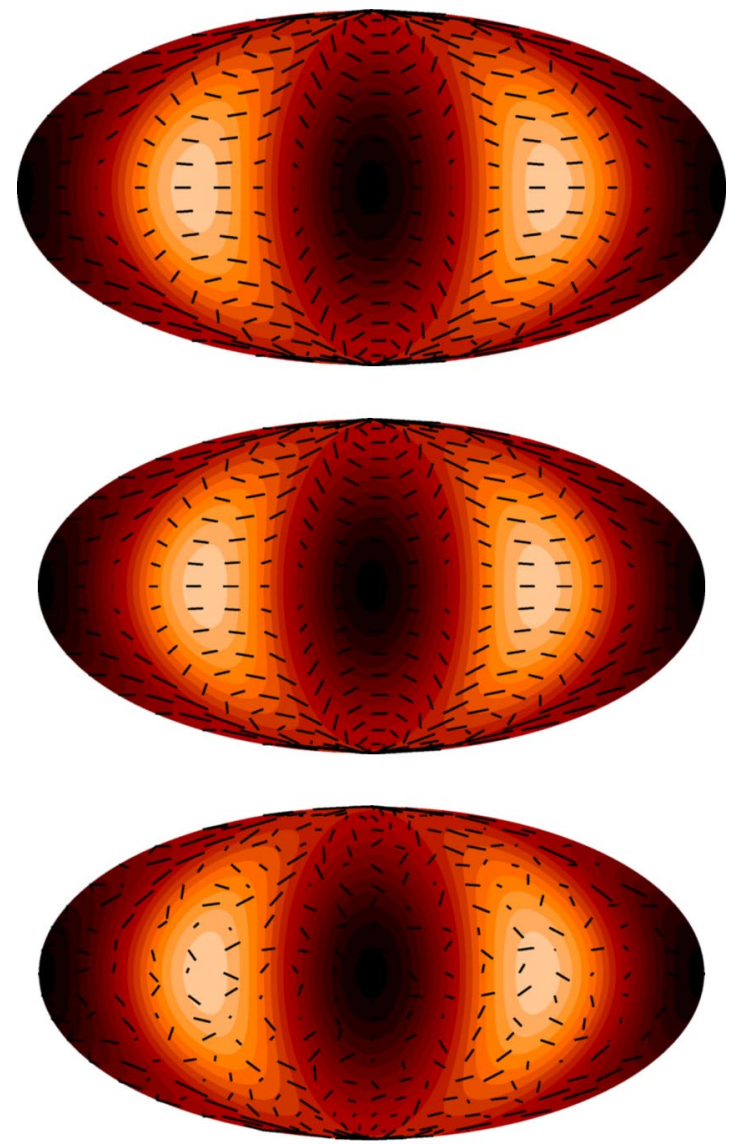

FIG. 1. (Color online only) The CMB polarization due to galaxy clusters. The polarization vectors give a representation of the expected polarization from a cluster at the corresponding location in the sky. The scale is such that the maximum length of the line corresponds to a polarization of $4.9 \tau \mu \mathrm{K}$. The background color represents the temperature quadrupole. The top plot shows the resulting polarization contribution due to scattering of the primordial temperature quadrupole alone. The middle map shows the total polarization contribution (primordial and kinematic) at $600 \mathrm{GHz}$; the total is clearly dominated by the primordial contribution. To highlight the kinematic polarization, in the bottom map, we arbitrarily increase its amplitude by a factor of 100 . Note that even at high frequencies where the kinematic quadrupole is increased due to its spectral dependence, given in Eq. (7), the primordial polarization still dominates the total contribution.

locities are uncorrelated; the correlation length of the velocity field of order $60 \mathrm{Mpc}$ correlates velocities within regions of $1^{\circ}$ when projected to a redshift of order unity. Such a correlation scale is much smaller than the all-sky maps we have considered in Fig. 1.

As shown in Fig. 1, even at high frequencies where the kinematic quadrupole is increased due to the spectral dependence, the primordial polarization still dominates the total contribution. This is also true when allowing for the fact that we may have underestimated the transverse velocity field; while the maximum primordial quadrupole related polarization remains at $4.9 \tau \mu \mathrm{K}$, the polarization associated with the kinematic quadrupole scales as $0.27 g(x)\left(\beta_{t} / 0.001\right)^{2} \tau \mu \mathrm{K}$. To get a comparable contribution at frequencies of the order 
of a few $100 \mathrm{GHz}$ with $g(x) \sim$ few, the transverse velocity field should involve magnitudes of order $1000 \mathrm{~km} \mathrm{sec}^{-1}$; such a high velocity is unlikely to be present in the large scale structure. The fact that primordial polarization dominates over the kinematic contribution even at high frequencies is useful for the present study since the reconstruction of the primordial quadrupole is not likely to be confusion limited.

To find the best-fit estimator $\mathcal{P}^{0}$ associated with $\mathbf{Q}$, and its variance, we calculate, for, say, one of the components of polarization,

$$
\frac{\delta}{\delta \mathcal{P}_{x}^{i}}\left\langle\left(P_{x}^{a}-\sum_{i=0}^{1} g^{i} \mathcal{P}_{x}^{i}\right)^{2}\right\rangle=0 .
$$

Here, $i=0$ denotes the $\mathbf{Q}$ contribution and $i=1$ denotes the $\mathbf{K}$ contribution. $g^{i}$ defines the spectral dependence of both effects; by definition $g^{0}=1$ as we consider variation of the kinematic quadrupole contribution relative to the thermal CMB. $g^{1}$ is given by Eq. (7).

Following Ref. [16], we find the foreground degradation factor $\left(F_{\mathrm{FD}}\right)$ as

$$
F_{\mathrm{FD}}=\left[\frac{1}{1-\left(g^{0} \cdot g^{1}\right)^{2} / g^{1} \cdot g^{1}}\right]^{1 / 2},
$$

where the dot product is defined as

$$
A \cdot B \equiv \sigma_{\mathcal{P}_{x}}^{(0) 2} \sum_{a, b=1}^{N_{\mathrm{ch}}} A_{a} C_{a b}^{-1} B_{b},
$$

with variance in the absence of the kinematic quadrupole contribution given by

$$
\sigma_{\mathcal{P}_{x}}^{(0) 2}=\frac{1}{\sum_{a=1}^{N_{\mathrm{ch}}}\left[C_{a b}^{x}\right]^{-1}} .
$$

Finally, the variance in the presence of the kinematic quadrupole contribution is given by

$$
\sigma_{\mathcal{P}_{x}}^{2}=\left(F_{\mathrm{FD}}\right)^{2} \sigma_{\mathcal{P}_{x}}^{(0) 2} .
$$

\section{B. Primordial quadrupole reconstruction}

We can calculate the expected errors in the primordial quadrupole reconstruction by considering the enhancement in the instrumental noise due to the separation of the contaminant kinematic quadrupole contribution. Under the assumption that the spectral dependence of the kinematic quadrupole, $g(x)$, is known, we can write the final variance of the determined quadrupole polarization as

$$
\sigma_{\mathcal{P}}^{2}=2\left(F_{\mathrm{FD}}\right)^{2}\left(\sum_{i=1}^{N_{\mathrm{ch}}} 1 / \sigma_{i}^{2}\right)^{-1} .
$$

Here, for simplicity, we have taken the instrumental noise covariance matrix to be diagonal with variance in each of the polarization components given by $\sigma_{i}^{2}$. The factor of 2 accounts for the two polarization components, $\left(P_{x}, P_{y}\right)$, with equal variance. For the case of equal variance in all channels, $\sigma=\sigma_{i}, \sigma^{2} \rightarrow 2\left(F_{\mathrm{FD}}\right)^{2} \sigma^{2} / N_{\mathrm{ch}}[16]$.

Given the uncertainty in the primordial polarization determination, Eq. (15), we can calculate the expected uncertainty in the rms quadrupole by considering Eq. (1). In Fig. 2, we show the redshift distribution of clusters expected from planned SZ surveys as calculated using the Press-Schechter [18] mass function based on analytic approaches in the literature [19]. Note that the selection functions of clusters in SZ surveys are essentially constant in mass over a wide range in redshift. An experiment such as the planned South Pole Telescope is likely to detect clusters down to a mass of $10^{14} \mathrm{M}_{\odot}$ [20]. Here, we assume a similar survey but roughly a factor of 2.5 increase in sky coverage with a total area of $\sim 10000 \mathrm{deg}^{2}$.

We bin the redshift distribution of these clusters in five bins between redshifts of 0 and 5 . We calculate the optical depth to scattering in each of these clusters by assuming constant gas mass fractions and converting the gas mass in each cluster to a number density of electrons under spherical arguments. More specifically, we follow the approach in Ref. [21] and describe the electron distribution, $n_{e}(r)$, within clusters based on hydrostatic arguments related to the cluster dark matter potential formed by the universal halo profile of Ref. [22]. The normalization of this density profile is set by requiring the gas to total mass ratio, $M_{\mathrm{gas}} / M_{\mathrm{tot}}$, of clusters to agree with the universal baryon fraction given by $\Omega_{b} / \Omega_{m}$; note that this condition sets the relative optical depths as a function of cluster mass. While most massive clusters have higher optical depths, they are less abundant. The ideal clusters for this study involve masses around $10^{14} \mathrm{M}_{\odot}$.

Note that for the present calculation involving cluster polarization, we only require knowledge of the gas distribution and not of the temperature structure; the latter is somewhat uncertain due to processes such as cooling and heating within clusters. To account for instrumental effects, mainly the finite resolution of polarization maps that can be made, we smooth the polarization profile from each cluster with a Gaussian beam of 5 arc min. This value is taken to be consistent with future experiments such as Planck as well as planned ground-based experiments with bolometer arrays. In addition to the profile discussed in Ref. [21], we also tested our calculations with profiles related to the $\beta$ model, $n_{e}(r)$ $\propto\left(1+r^{2} / r_{c}^{2}\right)^{-3 \beta / 2}$, but found consistent values. Therefore, we do not consider our choice of number density profile in individual clusters to be a major drawback when discussing potential detectability of the ISW signature with cluster polarization.

The optical depths, averaged over the beam size, range from 0.005 to 0.02 for individual clusters and typical polarization contributions, due to the temperature quadrupole, range from 0.02 to $0.1 \mu \mathrm{K}$. While one would require highly sensitive observations with an instrumental noise contribution below this level to detect the polarization contribution reliably towards an individual cluster, for the study suggested here, this is not necessary. Since we are attempting to make a 


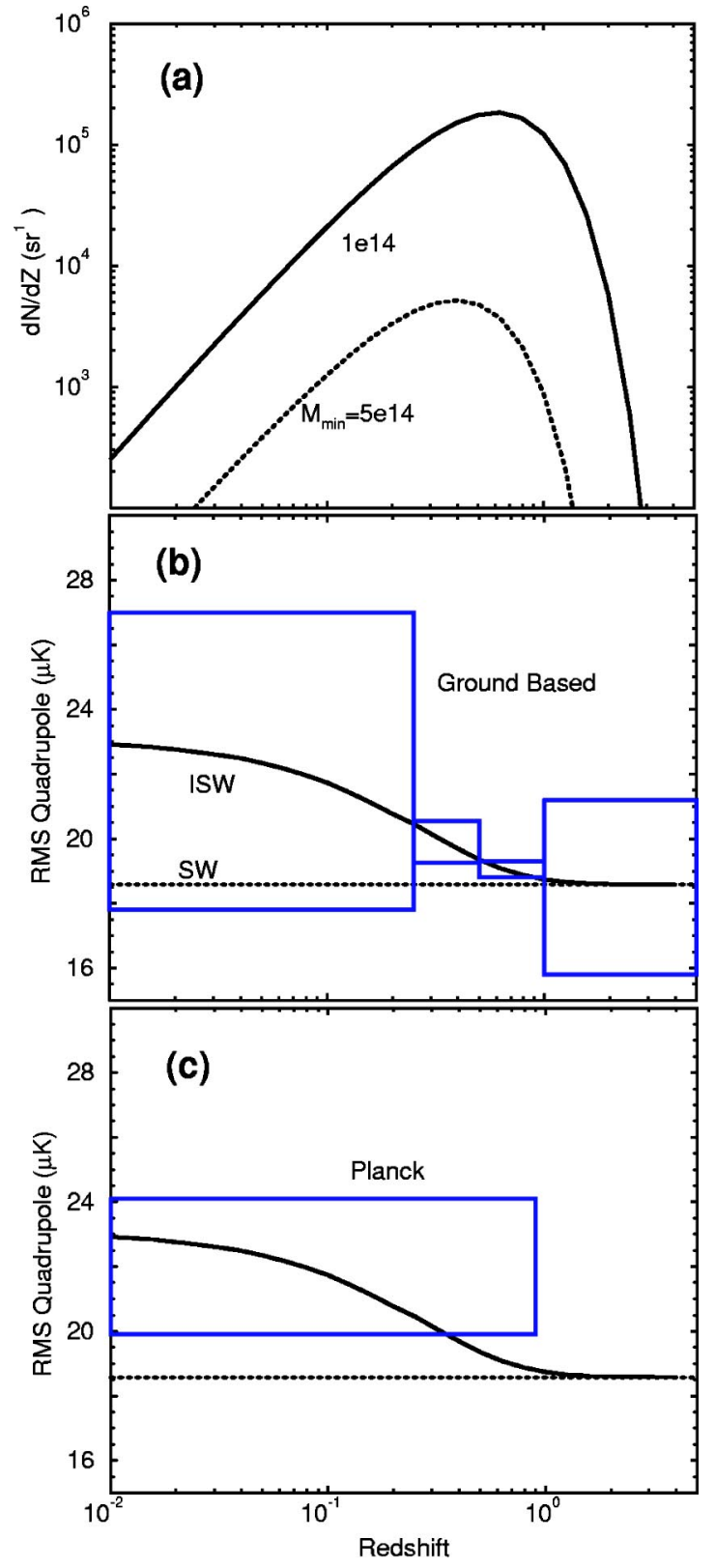

FIG. 2. (Color online only) The expected redshift distribution of galaxy clusters in SZ surveys (a). The upcoming surveys, such as the one planned with $4000 \mathrm{deg}^{2}$ observations with the South Pole Telescope, can probe mass limits down to $10^{14} \mathrm{M}_{\odot}$ and with the Planck surveyor down to $5 \times 10^{14} \mathrm{M}_{\odot}$. The rms temperature quadrupole as a function of redshift (b). The error bars illustrate the expected uncertainties in the reconstruction of the rms temperature quadrupole from follow-up multifrequency polarization observations of galaxy clusters with a catalogue down to $10^{14} \mathrm{M}_{\odot}$ and covering $10000 \mathrm{deg}^{2}$ (see text for details). In (c), the large single error illustrates the expected uncertainty with the whole-sky Planck cluster catalogue down to a mass limit of $5 \times 10^{14} \mathrm{M}_{\odot}$ and using Planck polarization observations of the same clusters.

statistical detection of the rms quadrupole, one can average over large samples of clusters widely distributed on the sky and the final variance in the rms quadrupole can be improved, in principle, by the number of individual clusters.

To consider the frequency separation of the cluster polar- ization contributions, we assume an experiment with observational frequencies at 30,100, 200 and $400 \mathrm{GHz}$ with a final polarization sensitivity of $1 \mu \mathrm{K}$ in each of the channels. The frequency degradation factor $\left(F_{\mathrm{FD}}\right)$ in this case is 2.07 . For polarization sensitive Planck channels, at 147, 217 and 545 and published sensitivities ranging from 10 to $560 \mu \mathrm{K}$, we determined a $F_{\mathrm{FD}}$ of 4.1. In general a low frequency channel at the Rayleigh-Jeans limit is required to reduce the degradation in noise, due to frequency cleaning, to factors of 2 and below.

Using $F_{\mathrm{FD}}$ for our four channel experiment and the redshift distribution of clusters, we can now determine how well the rms quadrupole can be established. We illustrate the expected rms quadrupole and errors around the top curve in Fig. 2. Here, we have taken a flat cosmological model with a matter density, in units of the critical density, $\Omega_{m}=0.35$, baryon density $\Omega_{b}=0.05$, dark-energy density $\Omega_{x}=0.65$ with an equation of state $w=-1.0$, a Hubble constant $h$ $=0.65$, and a spectral index of $n=1$ for primordial density perturbations. This corresponds to the usual case of a cosmological constant. We have normalized matter fluctuations to scales of $8 h^{-1} \mathrm{Mpc}$, with $\sigma_{8}=1.0$. For illustration purposes, we also show the expected rms temperature quadrupole for $w=0$; this corresponds to the case where dark energy behaves as matter, leading to an Einstein-de Sitter cosmology. In such a cosmology, $d / d r_{z}(G / a)=0$ [Eq. (2)] and there is no ISW contribution to the rms quadrupole.

We also make use of the Planck cluster catalogue as well as Planck polarization observations with expected sensitivities. The Planck SZ cluster catalogue is expected to detect clusters down to a mass limit of $5 \times 10^{14} \mathrm{M}_{\odot}$ [23]. While the number of counts per steradian is lowered (Fig. 2), on gains by the fact that the Planck catalogue is all sky. Still, due to the poor sensitivity of polarization observations, the reconstruction is noise dominated and no useful information can be obtained by binning the catalogue as a function of redshift. Thus, we combine all data in a single bin to estimate the rms temperature quadrupole. With Planck, one can determine the excess quadrupole, but it is unlikely that detailed information on its evolution can be established. We therefore suggest follow-up observations of the same cluster catalogue either through individual targeted observations or as part of a post-Planck polarization mission that may attempt to detect the signature of inflationary gravitational waves.

In estimating quadrupole reconstruction errors, we have ignored uncertainties associated with the determination of optical depth in each cluster. In the case of Planck data, if the optical depth can be established at the level of a few tens of percent or better, the final uncertainty in the temperature quadrupole reconstruction from polarization will be dominated by noise associated with polarization measurements and not in the determination of individual cluster optical depth; given current expectations based on cluster scaling relations, it is very likely that one can determine the optical depth to tens of percent or better in upcoming data.

For the suggested improved polarization experiment, the situation, however, is different. For the polarization noise there to dominate the final quadrupole reconstruction, optical depths to individual clusters should be known at the level of 
a few percent or better. Such a determination will require an improved understanding of cluster gas physics so that one can reliably convert the observed SZ thermal and kinetic temperature decrements to measurements of optical depth. During the Planck era, with the availability of multifrequency data, we expect that such a determination may be possible. Furthermore, potential studies with clusters, such as the one proposed here, motivate improvements in our understanding of cluster physics and we do not consider our inability to determine optical depths to be the limiting factor.

\section{DISCUSSION}

As illustrated in Fig. 2, a CMB temperature and polarization survey of $10000 \mathrm{deg}^{2}$ down to a fixed mass limit of $10^{14} \mathrm{M}_{\odot}$ can be used to reliably reconstruct the rms temperature quadrupole and its evolution. The temperature information is used to detect and catalogue clusters as well as to determine the optical depth to scattering. The same cluster sample is then followed up with multi-frequency polarization observations. In reconstructing the primordial temperature quadrupole, one is using these clusters as a tracer population in which each cluster individually provides information on the quadrupole.

The first attempt to perform a study similar to this will come from Planck's temperature and polarization observations. While Planck will provide an adequate size catalogue of clusters distributed over the whole sky, the poor sensitivity of polarization observations prevents one from extracting detailed information on the evolution of the temperature quadrupole. The Planck catalogue, however, can be used to potentially detect the excess of the temperature quadrupole rms at low redshifts due to the ISW effect.

For a more detailed detection of the ISW effect, including its redshift evolution, one needs an order of magnitude higher sensitivity polarization observations than Planck. An opportunity for such a study can be considered with respect to planned SZ surveys with telescopes such as the South Pole Telescope. If these upcoming observations can be made sensitive to polarization, the cluster catalogue detectable from such wide field SZ surveys can potentially be used for a more reliable reconstruction of the ISW signature. The advent of polarization sensitive bolometers suggest that such possibilities will be within reach over the next decade.

The suggested reconstruction here is beyond what was considered in Ref. [8]. Here, in addition to establishing the quadrupole, we have suggested the use of its evolution to directly observe the presence of the ISW effect. Since large angular scale temperature observations or cross correlations of the temperature with maps of the local universe cannot be used to establish the ISW effect reliably, cluster polarization observations provide an indirect method for this purpose.

The reconstruction, however, is subject to several confusions. Here, we have considered the confusion associated with the kinematic quadrupole contribution and have suggested the use of multifrequency polarization observations as a way to separate the two contributions. With adequate frequency coverage, this separation can be achieved with an increase in the variance of polarization noise by a factor of up to $\sim 2$.

In addition to the kinematic quadrupole polarization, galaxy clusters also produce polarization signals associated with two other mechanisms that generate a local quadrupole. These effects are due to the intrinsic quadrupole associated with double scattering effects within a single cluster. After the first scattering, the $\mathrm{CMB}$ acquires an local anisotropy both due to thermal and kinematic effects. Second scattering then produces polarization. The polarization associated with the thermal effect is proportional to $\tau^{2} k T_{e} / m_{e} c^{2}$. The kinematic double scattering induces a polarization signal proportional to $\tau^{2} \beta$. The frequency dependence of the polarization will be the same as that of the thermal or kinematic effect, respectively. Both second scattering effects are expected to be small due to their dependence on $\tau^{2}$. Additionally, for double scattering with a kinematic intrinsic quadrupole, when averaging over large samples, one expects $\beta \approx 0$ due to the randomness associated with the large scale velocity field. This will further suppress the contaminant signal. Including the double scattering associated with a thermal intrinsic quadrupole leads to biases in the rms quadrupole estimate of the order of a few percent in $\mu \mathrm{K}$. For unbiased estimates of the quadrupole, one can potentially account for this contaminant with prior knowledge of $\tau$ and $T_{e}$.

In addition to establishing the presence of the ISW effect reliably, the reconstruction is also useful for cosmological purposes. As is now well known, the ISW contribution, and thus the rms quadrupole, is strongly sensitive to basic properties of the dark energy such as its equation of state. Although not considered in this paper, the ISW contribution also varies significantly with clustering aspects of the dark energy component beyond the usual smooth assumption, such as in the generalized dark matter models of Ref. [24]. Considering a Fisher-matrix approach to the measurements shown in Fig. 2, assuming a cosmological model with the fiducial parameters listed earlier, we determined that one can extract the equation of state of the dark energy, $w$, with an uncertainty of $\sim 0.3$. Beyond estimates of cosmology, we suggest that in the future a reconstruction such as the one discussed here should be considered to establish the presence of the ISW effect and its evolution.

\section{ACKNOWLEDGMENTS}

We thank A. Challinor and I. Maor for useful comments on our paper. This work was supported in part by DOE DEFG03-92-ER40701, the Sherman Fairchild Foundation (A.C.), and Caltech (D.B.). A.C. thanks the Kavli Institute for Theoretical Physics (supported by NSF PHY99-07949) for hospitality while this work was completed. D.B. thanks the Astrophysics Group of the Cavendish Laboratory where parts of this work were carried out. 
[1] S. Chandrasekhar, Radiative Transfer (Dover, New York, 1960); N. Kaiser, Mon. Not. R. Astron. Soc. 101, 1169 (1983).

[2] W. Hu and M. White, New Astron. 2, 323 (1997).

[3] M. Zaldarriaga, Phys. Rev. D 55, 1822 (1997).

[4] R.A. Sunyaev and Ya.B. Zel'dovich, Mon. Not. R. Astron. Soc. 190, 413 (1980).

[5] S.Y. Sazonov and R.A. Sunyaev, Mon. Not. R. Astron. Soc. 310, 765 (1999).

[6] A. Challinor, M. Ford, and A. Lasenby, Mon. Not. R. Astron. Soc. 312, 159 (2000); E. Audit and J.F.L. Simmons, ibid. 305, L27 (1999).

[7] W. Hu, Astrophys. J. 529, 12 (2000); D. Baumann, A. Cooray, and M. Kamionkowski, astro-ph/0208511.

[8] M. Kamionkowski and A. Loeb, Phys. Rev. D 56, 4511 (1997).

[9] R.K. Sachs and A.M. Wolfe, Astrophys. J. 147, 73 (1967).

[10] R.G. Crittenden and N. Turok, Phys. Rev. Lett. 76, 575 (1996); S. Boughn, R. Crittenden, and N. Turok, New Astron. 3, 275 (1998); A. Kinkhabwala and M. Kamionkowski, Phys. Rev. Lett. 82, 4172 (1999).

[11] A. Cooray, Phys. Rev. D 65, 103510 (2002).

[12] C.L. Bennett et al., Astrophys. J. 436, 423 (1994).
[13] P. J. E. Peebles, The Large-Scale Structure of the Universe (Princeton University Press, Princeton, NJ, 1980).

[14] A. Cooray, W. Hu, and M. Tegmark, Astrophys. J. 540, 1 (2000).

[15] M. Tegmark, D.J. Eisenstein, W. Hu, and A. de Oliveira-Costa, Astrophys. J. 530, 133 (2000); L. Knox, Mon. Not. R. Astron. Soc. 307, 977 (1999).

[16] S. Dodelson, Astrophys. J. 482, 577 (1997).

[17] M. Kamionkowski, A. Kosowsky, and A. Stebbins, Phys. Rev. D 55, 7368 (1997); Phys. Rev. Lett. 78, 2058 (1997); M. Zaldarriaga and U. Seljak, Phys. Rev. D 55, 1830 (1997); U. Seljak and M. Zaldarriaga, Phys. Rev. Lett. 78, 2054 (1997).

[18] W.H. Press and P. Schechter, Astrophys. J. 187, 425 (1974).

[19] A. Cooray and R. Sheth, Phys. Rep. 372, 1 (2002).

[20] Z. Haiman, J.J. Mohr, and G.P. Holder, Astrophys. J. 553, 545 (2000).

[21] A. Cooray, Phys. Rev. D 62, 103506 (2000).

[22] J. Navarro, C. Frenk, and S.D.M. White, Astrophys. J. 462, 563 (1996).

[23] S.T. Kay, A.R. Liddle, and P.A. Thomas, astro-ph/0102352.

[24] W. Hu, Astrophys. J. 506, 485 (1998). 